\title{
Hematopoiesis in Regenerated Bone Marrow Within Hydroxyapatite Scaffold
}

\author{
ATSUSHI FUJITA, MAKOTO MIGITA, TAKAHIRO UEDA, REI OGAWA, YOSHITAKA FUKUNAGA, AND TAKASHI SHIMADA
}

Departments of Pediatrics [A.F., M.M., T.U., Y.F.], Biochemistry [A.F., M.M., T.S.], and Plastic and Reconstructive Surgery [R.O.], Nippon Medical School, Tokyo 113-8602, Japan

\begin{abstract}
We examined whether regenerated bone marrow (BM) with BM-derived stromal cells (BMSCs) on hydroxyapatite (HA) scaffolds can reconstitute the functional niche. Ly5.2 BMSCs on HA scaffolds were implanted s.c. onto the backs of Ly5.2 recipient mice. Lineage negative Ly5.1 BM cells expressing luciferase (luc) were i.v. administered into the recipient mice. Eight weeks after primary transplantation, secondary implantation was performed; the scaffolds removed from the first recipient mice were s.c. implanted into secondary recipient mice. Luc + cells were detected in the scaffolds for 6 mo after secondary implantation. Injection of G-CSF resulted in wide distribution of bioluminescence from the original scaffolds to the whole body. Even after removing the scaffolds from the secondary recipient mice, luc + cells were emitted by G-CSF stimulation, indicating that regenerated BM is capable of supporting hematopoietic stem cells (HSCs) and delivering HSCs to native BM in vivo. These data suggest that the functional niche is reconstituted at least partly and that regenerated BM on the scaffold may be used as a portable source of HSCs. (Pediatr Res 68: 35-40, 2010)
\end{abstract}

$\mathrm{H}$ ematopoietic stem cells (HSCs) are responsible for blood cell production throughout an individual's lifetime. Interaction of HSCs with their specific microenvironments, known as stem cell niches (1), is critical for maintaining the properties of stem cells, including the capacity to self-renew and the ability to differentiate into multiple lineages. During postnatal life, the bone marrow (BM) supports both selfrenewal and differentiation of HSCs in specialized microenvironmental niches. HSCs and hematopoietic progenitor cells (HPCs) are not randomly distributed in the BM (2) but instead are localized close to osteoblastic cells lining the endosteal surface [the endosteal niche (3-6)] and to sinusoidal endothelial cells within the perivascular area [the vascular niche $(7,8)]$. Previous studies have reported that HSCs properties are maintained in the osteoblastic niche and that HSCs differentiate here before mobilizing to the vascular niche. In the vascular niche, hematopoietic stem and progenitor cells (HSPCs) differentiate and mobilize to the peripheral circulation (9).

Limited success has been achieved with several combinations of cytokines (10) explored to expand HSPCs ex vivo, and stromal cells $(11,12)$ and osteoblasts $(13)$ that are components

Received January 5, 2010; accepted April 2, 2010.

Correspondence: Makoto Migita, M.D., Ph.D., Department of Pediatrics, Nippon Medical School, 1-1-5 Sendagi, Bunkyo-ku, Tokyo 113-8602, Japan; e-mail: mmigita@nms.ac.jp

Supported in part by Kawano Masanori Memorial Foundation for Promation of pediatrics. of the niche are reported to be able to promote the survival, proliferation, and differentiation of HSPCs in vitro. Moreover, some degree of efficacy has been demonstrated for fibronectin, nanofiber scaffolds (14), and 3D scaffolds (15) in the expansion of HSPCs. However, these attempts could not maintain HSPC properties in the long term. Therefore, the possibility of using regenerated BM in a hydroxyapatite (HA) scaffold in vivo as a functional niche has been studied for maintenance, proliferation, and expansion of HSPCs.

Various synthetic products have been developed and used as bone substitutes for bone regeneration. Of all the materials available, calcium phosphate ceramics have good biocompatibility and osteoconductivity, and they give good results as bone substitutes and scaffolds. HA (16), a calcium phosphate ceramic (17), shows these properties and is widely used in clinical applications $(18,19)$. After BM-derived stromal cells (BMSCs) are cultured on HA scaffolds, the scaffolds with attached cells induce not only bone but also BM-like tissues at extraosseous sites $(16,20,21)$. The anatomical architecture of BM-like tissues contains osteoblast-like cells and blood vessels $(16,20)$. However, it is not known whether BM-like tissues function comparably to native $\mathrm{BM}$ as a BM niche for HSPCs.

The principal aim of our study was to evaluate whether the BM-like tissues within the scaffolds (regenerated BM) have the same ability as the native BM to maintain HSPC properties and contribute to hematopoiesis in vivo.

\section{MATERIALS AND METHODS}

Mice. C57B1/6 (Ly5.2) mice, green fluorescent protein (GFP) transgenic mice [C57BL6 TgN (act-EGFP) OsbC14-Y01-FM131], and C57B1/6-CD45.1 (Ly5.1) congenic mice were purchased from Saitama Experimental Animals Supply (Saitama, Japan). Animal care in our laboratory was in accordance with the Nippon Medical School guidelines for animal and recombinant DNA experiments.

The HA scaffold. We used the HA scaffold (NEOBONE; MMT, Co.) (22) in this study. It was produced from a slurry of HA by the "foam-gel technique," and has a systematic arrangement of spherical uniform pores. It was sintered at $1200^{\circ} \mathrm{C}$ with the following characteristics: porosity, $75 \%$; mean porous diameter, $150 \mu \mathrm{m}$; interconnective pore hole, 10-90 $\mu \mathrm{m}$ (mean $40 \mu \mathrm{m}$ ). Cylindrical brocks $5 \mathrm{~mm}$ in diameter and $2 \mathrm{~mm}$ in length were prepared for this study.

Abbreviations: BM, Bone marrow; BMSCs, Bone marrow derived stromal cells; HA, Hydroxyapatite; luc, Luciferase; HSCs, Hematopoietic stem cells; HPCs, Hematopoietic progenitor cells; HSPCs, Hematopoietic stem and progenitor cells; Ly5.2, C57Bl/6; GFP, Green fluorescent protein; Ly5.1, C57B1/6-CD45.1; Lin, Lineage negative; PB, Peripheral blood; IVIS, In vivo imaging system 
Isolation of BMSCs. BMSCs were harvested from the femurs and tibias of 5 to 8-wk-old GFP transgenic mice. Whole BM cells were obtained under sterile conditions by flushing the BM cavity with DMEM (GIBCO BRL, Grand Island, NY) in a 1-mL syringe with a 23 -gauge needle. The resulting cell suspension was plated and cultured in DMEM containing $10 \%$ fetal bovine serum (FBS) at $37^{\circ} \mathrm{C}$ under a $5 \% \quad \mathrm{CO}_{2}$ atmosphere. The culture medium was replaced every 3-4 d, and all nonadherent cells were then removed for $14-21 \mathrm{~d}$. When confluent, the adherent cells were trypsinized and passaged approximately at a 1:3 split. BMSCs were expanded until passage 2 or 3 before they were seeded on the scaffolds.

Regeneration of BM in the HA scaffold. The GFP-positive BMSCs were harvested and 1 to $4 \times 10^{6}$ cells were trypsinized and replated onto a HA scaffold. Cells were incubated in an osteogenic medium (DMEM, 10\% FBS, $0.1 \mathrm{M}$ dexamethasone, $50 \mathrm{M}$ ascorbic acid-2-phosphate, and $10 \mathrm{mM}$ betaglycerophosphate) for $3 \mathrm{~d}$, to induce osteogenesis. GFP luminescence of scaffolds was detected by fluorescence microscopy to confirm that BMSCs had attached. Ly 5.2 mice ( 8 to 12 wk old) were anesthetized with pentobarbital sodium, and 1 to 6 scaffolds containing BMSCs were transplanted s.c. onto the back of each mouse.

Isolation of HSPCs. BM was collected from the femurs and tibias of Ly 5.1 mice (6 to $10 \mathrm{wk}$ old), Isolated BM cell suspension was centrifuged at $400 \times \mathrm{g}$ for $5 \mathrm{~min}$, after which the cell pellet was resuspended in $5 \mathrm{~mL}$ of red cell lysing buffer ( $155 \mathrm{mM} \mathrm{NH} 4 \mathrm{Cl}, 10 \mathrm{mM} \mathrm{KHCO} 3,0.1 \mathrm{mM}$ EDTA) for 10 $\mathrm{min}$ at room temperature to lyse the erythrocytes. The remaining cells were washed twice and filtered through a 40-mm filter (Becton Dickinson, Franklin Lakes, NJ). BM cells were labeled with a cocktail of biotinylated rat antimouse antibodies: B220, macrophage antigen-1 (Mac-1), Gr-1, CD4, CD8, CD3, CD5, and Ter119. Lineage negative (Lin-) Ly 5.1 positive (Ly5.1+) cells were isolated using auto magnetic-activated cell separation (auto MACS; Miltenyi Biotec).

Transduction of lentiviral vector to Lin - Ly 5.1 + BM cells. Lin- Ly 5.1 BM cells were transduced with a lentiviral vector containing the luciferase (luc) gene. The pCL20c MSCV-luc, modified from cPL20c MSCV-GFP (designated SJ1) (23), is a lentiviral vector based on HIV-1 that transduces the firefly luc gene. The luc gene was cut from PicaGene Promoter Vector 2 Sequence (PGV-P2) purchased from TOYO B-Net (Tokyo, Japan). Multiplicities of infection (MOIs) were determined in proliferating HeLa cells. Lin - Ly 5.1+ BM cells $\left(1.0 \times 10^{6}\right)$ were transduced with fresh lentiviral vector supernatant at an MOI of 75 in $2 \mathrm{~mL}$ of serum-free expansion medium (SFEM; Stem Cell tech, VERITAS, Tokyo, Japan) with $2 \mu \mathrm{L}$ of $100 \mu \mathrm{g} / \mathrm{mL}$ mouse stem cell factor (mSCF; Amgen, Thousand Oaks, CA) in 6-well plates. After $14 \mathrm{~h}$, nonadherent BM cells were harvested by washing twice with PBS.

$B M$ transplantation of Lin - luc + cells into primary recipient mice with implanted scaffolds. Between 8 and 12 wk after implantation of scaffolds, recipient mice with implanted scaffolds were lethally irradiated with $950 \mathrm{cGy}$ $2 \mathrm{~h}$ and were i.v. infused with $4.0 \times 10^{5}$ or $4.0 \times 10^{6} \mathrm{Lin}-$ luc + Ly $5.1+$ cells via the tail vein.

Implantation of scaffolds to secondary recipient mice. Between 8 and 12 wk after BM transplantation, secondary implantation was carried out to evaluate scaffold function. First, scaffolds including luc + Ly $5.1+$ cells were removed from primary recipient mice. Wild-type Ly 5.2 recipient mice were lethally irradiated with 950 cGy $2 \mathrm{~h}$ before secondary s.c. implantation with one to six pieces of scaffold. As the number of HSPCs in the scaffolds was insufficient for reconstitution, cells were cotransplanted with $3 \times 10^{5}$ of unfractionated and nontransduced Ly 5.2 host BM cells. In addition, to evaluate the reconstitution ability of s.c. implanted HSPCs, we also carried out secondary s.c. implantation of femurs from primary recipient mice with the same implantaion conditions, as a positive control. To assess whether the regenerated $\mathrm{BM}$ has the ability to support HSCs and to deliver HSCs to the native $\mathrm{BM}$ in vivo, mice were injected with recombinant human G-CSF (Filgrastim, Amgen, Thousand Oaks, CA, $250 \mu \mathrm{g} / \mathrm{kg} / \mathrm{d}$, every $12 \mathrm{~h}$, s.c. for $1-4$ d) in PBS supplemented with $0.1 \%$ low endotoxin BSA (BSA, Sigma Chemical Co., St. Louis MO). In vivo imaging was performed with an in vivo imaging system (IVIS) system (Xenogen, Alameda, CA; further details below) and peripheral blood (PB) was harvested.

Finally, the scaffolds were removed from the secondary recipient mice to determine whether HSCs derived from the regenerated BM in the HA scaffold had been engrafted in the recipient's native BM. Initially, the scaffolds were removed from the secondary recipient mice 5 mo after secondary implantation. G-CSF was s.c. injected into these mice after a further $7 \mathrm{mo}$, followed by in vivo imaging with the IVIS system.

Histologic analysis. After removal, scaffolds were visually observed and histopathologically evaluated. Scaffolds with surrounding tissues were fixed for $4 \mathrm{~d}$ and decalcified for $7 \mathrm{~d}$ with $4 \%$ (wt/vol) paraformaldehyde and $7 \%$ $(\mathrm{wt} / \mathrm{vol})$ formic acid. Samples were embedded in paraffin and sectioned with a microtome. Histopathological evaluations of the specimens were performed using hematoxylin-eosin (HE) staining.
In vivo bioluminescence imaging analysis. In vivo optical imaging was performed with a prototype IVIS 100 bioluminescence/fluorescence optical imaging system (IVIS; Xenogen, Alameda, CA) between 8 and 12 wk after primary BM transplantation. Before imaging, luciferin (Promega) was administered at a dose of $150 \mathrm{mg} / \mathrm{kg}$ i.p. At the time of imaging, animals were placed in a light-tight chamber and luminescent images were captured with 1 or 2 min exposure times by an IVIS employing a cooled charge-coupled device camera. The resulting images were automatically superimposed by software to facilitate identification and location of any optical signal in the mouse. Optical images were displayed and analyzed with the Igor (WaveMetrics, Lake Oswego, OR) and IVIS Living Image (Xenogen) software packages. Optical signal was expressed as photon flux, in units of photons/s.

Flow cytometric analysis. Flow cytometry was used to quantify the differentiation of hematopoietic cells in the PB, BM, and implanted scaffolds of transplanted mice. Mononuclear cells (MNCs) in the PB and BM cells were harvested using red cell lysis buffer. The scaffolds were ground in a mortar with PBS to isolate MNCs. After washing, suspensions were filtered through a $40-\mu \mathrm{m}$ filter to remove large particles, and low-density cells $\left(<1.083 \mathrm{~g} / \mathrm{cm}^{3}\right)$ were isolated by discontinuous density centrifugation using histopaque 1083 (Sigma Chemical Co.). Cells in PB, BM, and scaffolds were labeled with leukocyte antibodies (Ly5.1-FITC or Ly5.1-biotin; Pharmingen), lineage antibodies (B220-PE, CD3e-APC, CD4-PE, CD8a-APC, Mac-1-APC, and Gr-1-PE; Pharmingen), and stem cell markers (Sca-1-PE and c-kit-APC; Pharmingen), followed by analysis with FACS Calibur (Becton Dickinson).

\section{RESULTS}

\section{Structure and cellular composition of implanted scaffolds.} The experimental design is shown in Figure 1. The HA scaffold were incubated with BMSC from GFP + transgenic mice [Fig. 1 (1)]. After s.c. implantation of the in vitrocultured scaffolds to recipient mice [Fig. 1 (2)], the appearance and cellular composition of the scaffold was investigated (Fig. $2 C$ and $D$ ). Many vessels were observed on and surrounding the scaffolds (16) removed from recipient mice. This increased vacularity caused the scaffolds to turn red (Fig. $2 C$ ). In the scaffolds, bone matrix and BM-like tissue including a variety of blood cells and small vessels were confirmed on microscopy, as previously described (16) (Fig. 2D).

\section{(1) GFP+ BMSC on HA Scaffolds}

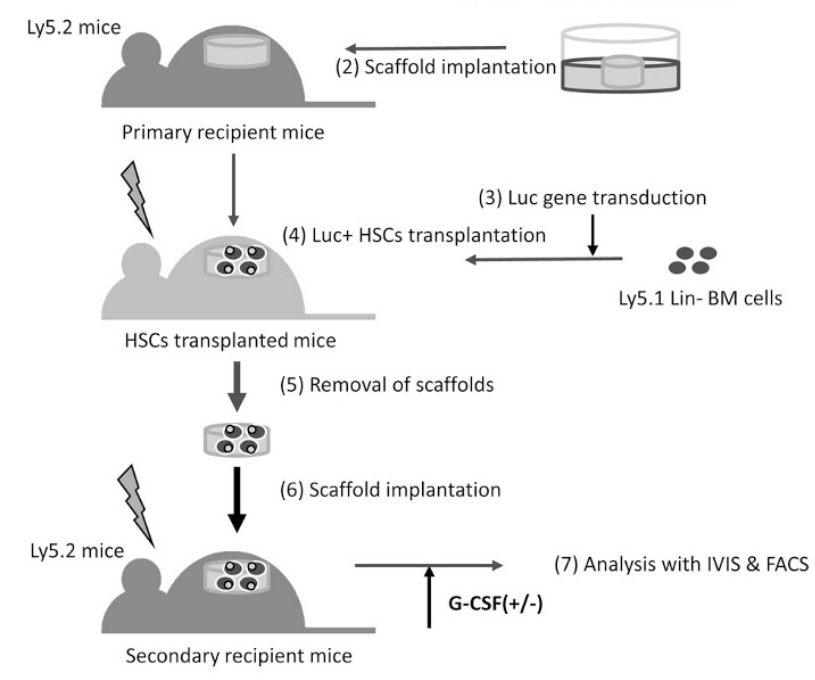

Figure 1. Experimental design. The HA scaffolds were incubated with BMSCs from GFP+ transgenic mice (1) and were s.c. implanted on to the back of primary recipient Ly5.2 mice (2). Lin- Ly.5.1 + BM cells were transduced with a lentiviral vector expressing the luc gene (3) and i.v. transplanted to the primary recipient mice with scaffolds after lethal irradiation (4). The scaffolds from the HSCs transplanted mice were removed (5) and implanted to secondary recipient mice (6). The secondary recipient mice were further characterized (7). 

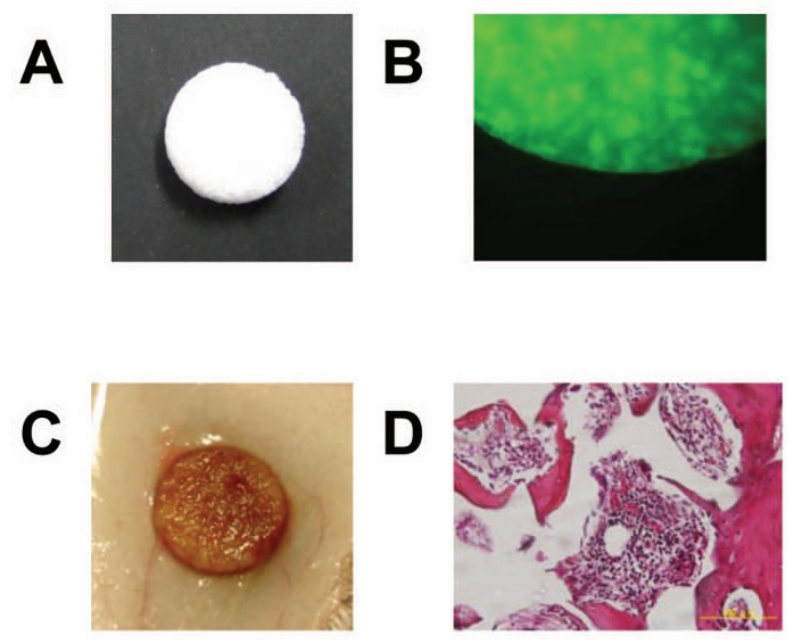

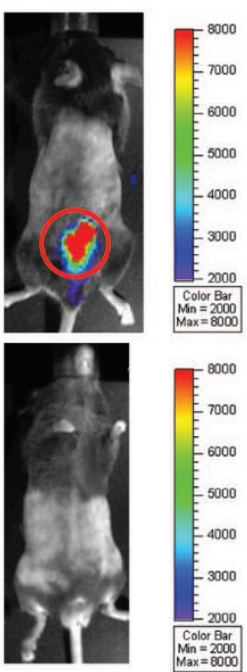

Figure 2. Characterization of the HA scaffolds and the recipient mice. $A$, HA scaffold cylinder (diameter $=5 \mathrm{~mm}$, length $=2 \mathrm{~mm}$ ) in this study. $B$, GFP luminescenne of scaffolds that BMSCs had attached was detected by fluorescence microscopy before implantation. Gross appearance $(C)$ and HE staining $(D)$ of the scaffold isolated from the primary recipient mice. Scale bar: $100 \mu \mathrm{m}$. Distribution of luc + HSCs in recipient mice was examined with an IVIS system $(E-H)$. Ventral view imaging of a primary recipient mouse was conducted $5 \mathrm{~d}(E)$ and dorsal view imaging of a primary recipient mouse was conducted $14 \mathrm{~d}$ $(F)$ after HSCs transplantation. Dorsal $(G)$ and ventral $(H)$ view whole body bioluminescence imaging of a secondary recipient mouse was measured $14 \mathrm{~d}$ after scaffold implantation. Red circles in $(F)$ and $(G)$ represent the implanted scaffold.
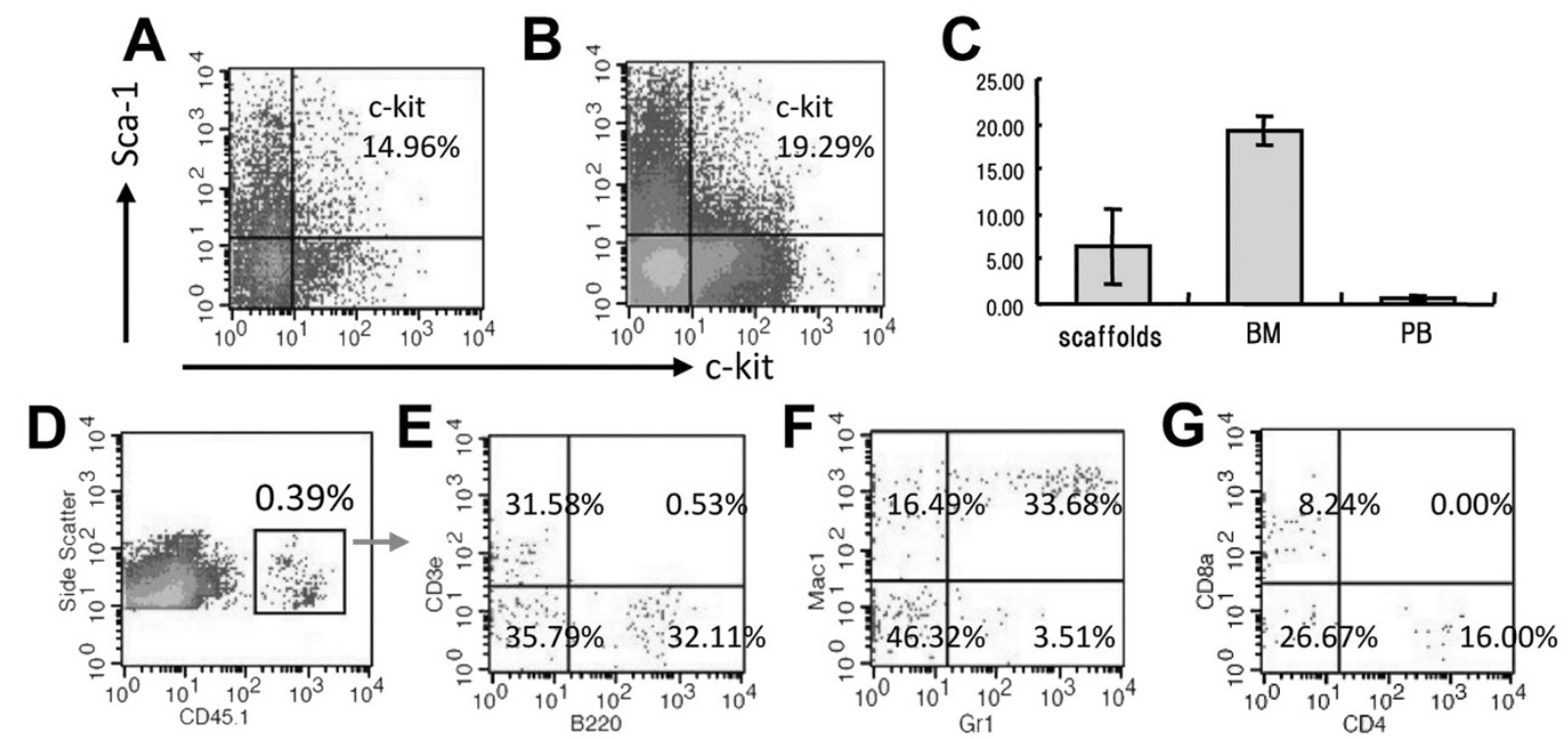

Figure 3. FACS analysis of hematopoietic cells in secondary recipient mice. Expression of c-kit and Sca-1 were analyzed on cells in implanted scaffolds (A) and BM $(B) 2$ mo after scaffold implantation. $(C)$ The ratio of c-kit + cells were compared and data are plotted as the mean $( \pm$ SD) in three groups, cells in scaffold $(6.38 \pm 4.25 \%, n=8), \mathrm{BM}(19.29 \pm 1.59 \%, n=5)$, and $\mathrm{PB}(0.55 \pm 0.30 \%, n=3)$. Ly5.1+ donor cells were detected in PB fraction from secondary recipient mice after G-CSF stimulation at 8 mo after scaffold implantation $(D)$. The differentiations of donor cells were further studied for expression of B220 and CD3e $(E)$, Gr1 and $\operatorname{Mac} 1(F)$, and CD4 and CD8 $(G)$.

\section{Distribution of luc + donor cells in primary transplanta-} tion. Lin- Ly5.1+ BM cells were transduced with a lentiviral vector containing the luc gene and transplanted into Ly5.2 mice with implanted scaffolds [Fig. 1 (3 and 4)]. Distribution of luc + donor cells in primary recipient mice was assessed on d 1, 3, 5, 7, 10, and 14 after BM transplantation by IVIS. Bioluminescence was detected as early as $24 \mathrm{~h}$ after transplantation, and the strongest signal was observed in the spleen. Bioluminescence gradually became distributed throughout the whole body (24) in $5 \mathrm{~d}$ (Fig. 2E). On the 14th d after transplantation, bioluminescent intensity was almost as strong at the vertebrae as at the scaffold, and this phenomenon continued until 2 mo after transplantation (Fig. 2F).

Distribution of luc + cells engrafted in scaffolds in secondary recipient mice. Eight weeks after primary transplantation, the scaffolds were removed from the first recipient mice [Fig. 1 (5)] and s.c. implanted into secondary recipient mice [Fig. 1 (6)]. Bioluminescent signals of luc + hematopoietic cells in implanted mice were detected only at the scaffolds and had not spread to other areas of the body by the 14th $\mathrm{d}$ after secondary implantation(Fig. $2 G$ and $H$ ). Bioluminescence levels in the supine position, measured as total photon flux 
(photon/s), was higher in mice implanted with multiple numbers of scaffolds when compared with those receiving one scaffold. No significant difference was observed in scaffold bioluminescence, irrespective of whether $4.0 \times 10^{5}$ Lincells or $40.0 \times 10^{5}$ Lin - cells were given in HSCs transplantation. Secondary recipient mice implanted with femurs showed stronger bioluminescence at very similar sites to mice implanted with scaffolds. Moreover, Ly 5.1+ donor cells were detected in PB (data not shown).

Immature hematopoietic cells engrafted in scaffolds. MNCs isolated from the PB, BM, and implanted scaffolds were immunostained and analyzed by flow cytometry. Immature hematopoietic cells were detected in scaffolds after transplantation (Fig. 3). The proportion of immature c-kit + cells in scaffolds varied across the mice (2.32-14.96\%). The proportion of c-kit + cells in the scaffolds was significantly greater than that in PB but lower than that in BM (Fig. 3C). Furthermore, $2.13 \%(n=3, \mathrm{SD}= \pm 1.51)$ of cells in scaffolds were Ly 5.1 positive between 6 and 8 mo after secondary implantation. These results indicate that the BM-like tissues in scaffolds can home, lodge, and engraft HSPCs comparably with the native BM microenvironment.

Behavior of Ly 5.1+luc+ cells after G-CSF treatment in secondary recipient mice. To further evaluate the function of implanted scaffolds, the secondary recipient mice were injected with G-CSF. Mobilization of Ly 5.1+ luc + HSPC stimulated with G-CSF were observed using IVIS analysis. After the injection of G-CSF for $4 \mathrm{~d}$, bioluminescence intensity increased and donor-derived positive cells were localized widely in recipient mice (Fig. 4A). The same finding was observed in the secondary recipient mice implanted with donor femur bone containing BM (Fig. 4B). Bioluminescent signals in the recipient mice were enhanced until the 15 th $d$ after G-CSF injection, and positive cells were observed in the body until the 50th d (Fig. 4C).

FACS analysis of PB after G-CSF stimulation showed that $0.39 \%$ of PB cells were Ly5.1 positive after 8 mo of secondary transplantation (Fig. 3D). At the same time point, $0.07 \%$ and $3.17 \%$ of cells were Ly 5.1 positive in femurs and in scaffolds. The proportion of c-kit + cells was $20.45 \%$ in BM and $14.96 \%$ in scaffolds. In addition, the proportion of Lin- cells was $8.35 \%$ in BM and $9.02 \%$ in scaffolds. All leukocyte subsets of Ly5.1+ were detected in PB, including CD4+ and CD8 $+\mathrm{T}$ cells, B220+ B cells, and Gr1+ Mac-1+ granulocytes. These results indicate that engrafted HSPCs in scaffolds have the long-term abilities of maintenance and differentiation.

Finally, the scaffolds were removed from the secondary recipient mice, and the resulting mice without regenerated $\mathrm{BM}$ were stimulated with G-CSF. Bioluminescent signals of luc+ hematopoietic cells became detectable in the whole body, indicating that luc + cells were emitted from the native BM of recipient mice (Fig. 4D).

\section{DISCUSSION}

Recent studies indicate that both BM niches, the endosteal niche and the vascular niche, are essential to renew and differentiate HSCs and HPCs and to mobilize HSPCs to the
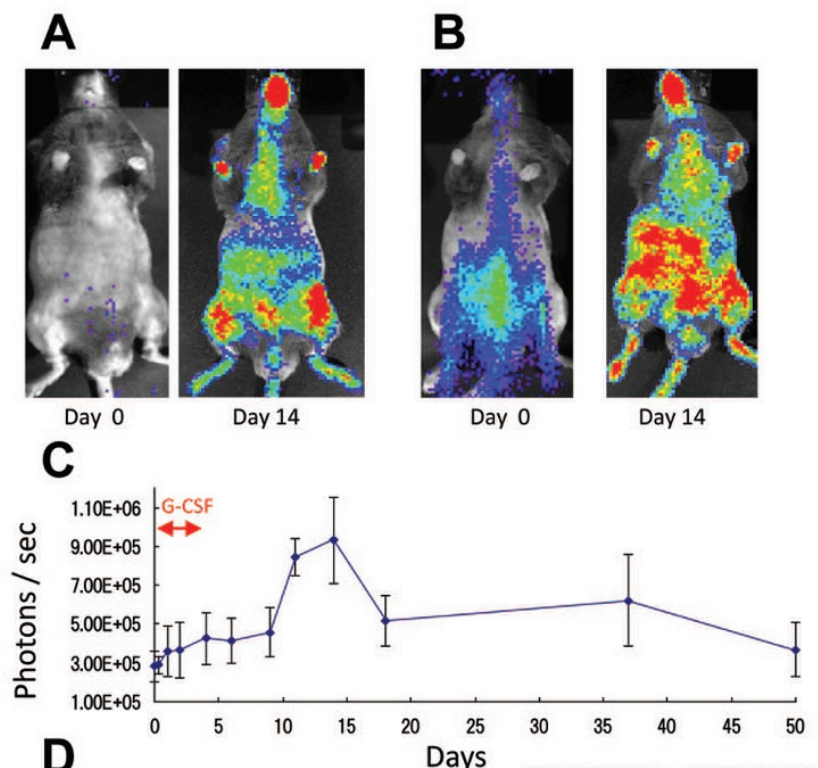

The scaffold was removed from the recipient
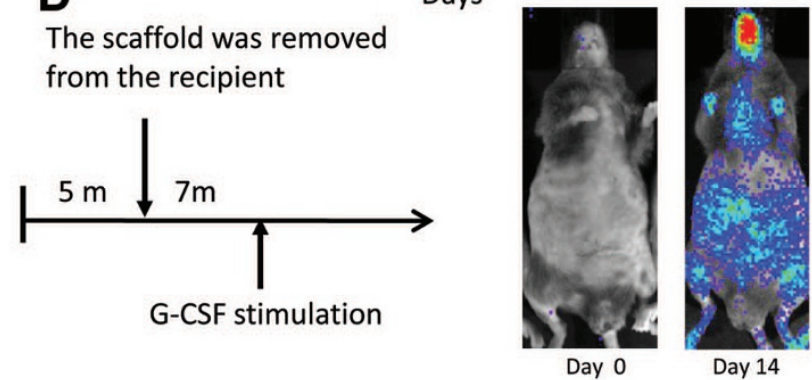

Figure 4. Mobilization of luc + transplanted cells in secondary recipient mice after G-CSF stimulation. Mice were implanted with scaffolds $(A)$ or femurs $(B)$, as a positive control, from luc + HSCs transplanted mice and were injected with GCS-F for $4 \mathrm{~d}$ at 3 or more months after scaffold implantation. Ventral view whole imaging was measured at d 0 and d 14 after GCS-F injection. $C$, Kinetics of luc + donor cells after G-CSF injection. Data are plotted as the mean $( \pm \mathrm{SD})$ for the secondary recipient mice $(n=5) . D$, Engraftment of luc + HSCs from implanted scaffolds into BM. Scaffolds were removed from the secondary recipient mice 5 mo after implantation. After 7 mo, the recipient mice without scaffolds were injected with G-CSF and ventral view whole imaging was assessed 0 and $14 \mathrm{~d}$ after G-CSF injection.

peripheral circulation $(9,25)$. Osteoblasts are essential components of the functional microenvironment for hematopoiesis in $\mathrm{BM}$ and are themselves a heterogeneous population $(3,4)$. Although mature osteoblasts contribute to bone formation, some immature osteoblastic cells that express $\mathrm{N}$-cadherin on the endosteal surface are currently thought to function as the niche cells for HSCs in the BM (4), and HSCs attached to these cells are mostly maintained in quiescence (6). Moreover, calcium ion levels in the niche are critical for migration and attachment of HSCs to the endosteal surface (26).

The vascular niche, which consists of endothelial cells, is also functionally required for hematopoiesis in vivo $(7,27)$. However, the type of cells forming the vascular niche remains unclear. Although the populations of HSCs and immature HPCs reside in the center of the BM, mature HPCs exist closer to small vessels in the BM. Thus, it is plausible that the endosteal niche provides a quiescent environment for HSCs maintenance and that the vascular niche offers an alternative niche for mobilized stem cells and promotes proliferation and further differentiation or maturation into the circulatory system. 
We report three novel findings in this study. First, the BM-like tissues regenerated in scaffolds have the ability to elicit HSPCs mobilization. Second, HSPCs can engraft in regenerated $\mathrm{BM}$, and hematopoietic cells were emitted from the G-CSF-stimulated secondary recipient mice. Finally, the BM-like tissues have the ability to maintain HSPCs and support their differentiation for as long as $8 \mathrm{mo}$. These findings lead us to conclude that the BM-like tissues in scaffolds play an important role in the support and maintenance of hematopoiesis and native BM. Hence, scaffolds have the function and the anatomical architecture of BM.

Histologic studies have shown that scaffolds consist of bone matrix and BM-like tissue including various kinds of blood cells and small vessels $(16,18)$. It is particularly important that complex BM components are histologically regenerated in these scaffolds. Several attempts using combinations of cytokines (10), stromal cells (11), osteoblasts (13), nanofiber scaffolds (14), and 3D scaffolds (15) have been made to support, regulate, and expand HSPCs. Although recent studies have suggested a functional classification of BM niches into two kinds, the endsteal (4) and the vascular (8), it seems likely that native $\mathrm{BM}$ in vivo is more complex and has longer term functions than those currently identified because actual BM is composed of many types of cells. The present findings support the notion that regenerated BM in the HA scaffold in vivo, which contains natural and living components can more efficiently support HSCs.

In the primary transplantation model, the bioluminescence of luc + cells in scaffolds was detected as strong as that of the femurs as positive control between the 14th and the 21st dafter transplantation. In addition, the proportion of c-kit + immature cells was significantly higher in scaffolds than in PB. These findings strongly support the view that scaffold provides an environment for HSPC homing, lodging, and engraftment that is comparable with that of native BM. However, the proportion of c-kit + cells in scaffolds varied in individual cases. To assess whether luc + Ly5.1+ donor cells in scaffolds can maintain HSPCs in the long term, scaffolds including luc + Ly5.1+ donor cells were secondary implanted into wild-type mice. We expected that donor derived luc + HSPCs expanded from scaffolds to the whole body and were detected for a considerable period. However, unexpectedly, these cells could not be detected in whole body using IVIS unless G-CSF was injected. We assume that the main reason why luc + Ly5.1+ cells were not detectable is that there were insufficient HSPCs in scaffolds to be detected with IVIS. However, we can at least show that hematopoietic function was as good in regenerated $\mathrm{BM}$ as in the native $\mathrm{BM}$ because luc + cells were emitted from the native BM of recipient mice without regenerated BM (Fig. $4 D$ ). This finding suggests that this regenerated $\mathrm{BM}$ has the ability both to maintain HSCs and mobilize and deliver HSCs from regenerated $\mathrm{BM}$ to the recipient's native $\mathrm{BM}$.

We showed that engrafted HSPCs in scaffolds have the long-term ability to support HSPCs maintenance and differentiation; this was evident in one mouse for $8 \mathrm{mo}$. Moreover, engrafted HSPCs were maintained in scaffolds in some mice for 6 mo after secondary implantation. In addition, for 3 or more months, bioluminescence of scaffolds continued in all mice, and engrafted immature cells were maintained in scaffolds. These results confirm that scaffolds have the ability to maintain HSCs, considering the life span of mature hematopoietic cells.

Our results suggest new treatments might be possible for fibrotic BM and hematopoietic diseases such as idiopathic myelofibrosis, osteopetrosis, and anaplastic anemia. To avoid pathologic immune responses and reduce invasive treatment, the use of autologous tissue may be preferable to BMSCs. We have already reported BM regeneration using adipose-derived stem cells (16), and other mesenchymal stem cells may be useful. Because BM has been compared with alternative hematopoietic tissues, such as fetal aorta-gonado-mesonephros, placenta, liver, and spleen, we expect that analyzing differences between the regenerated and native BM may provide new insights into hematopoiesis. The regeneration of the hematopoietic system using scaffolds may be useful in the future as an ex vivo means of supplying and expanding all types of blood cells.

Acknowledgments. We thank Kyowa Hakko Kirin Co., Ltd., for kindly providing G-CSF, and MMT Co., Ltd., for providing hydroxyapatite scaffold, NEOBONE.

\section{REFERENCES}

1. Schofield R 1978 The relationship between the spleen colony-forming cell and the haemopoietic stem cell. Blood Cells 4:7-25

2. Nilsson SK, Johnston HM, Coverdale JA 2001 Spatial localization of transplanted hemopoietic stem cells: inferences for the localization of stem cell niches. Blood 97:2293-2299

3. Calvi LM, Adams GB, Weibrecht KW, Weber JM, Olson DP, Knight MC, Martin RP, Schipani E, Divieti P, Bringhurst FR, Milner LA, Kronenberg HM, Scadden DT 2003 Osteoblastic cells regulate the haematopoietic stem cell niche. Nature 425:841-846

4. Zhang J, Niu C, Ye L, Huang H, He X, Tong WG, Ross J, Haug J, Johnson T, Feng JQ, Harris S, Wiedemann LM, Mishina Y, Li L 2003 Identification of the haematopoietic stem cell niche and control of the niche size. Nature 425:836-841

5. Adams GB, Scadden DT 2006 The hematopoietic stem cell in its place. Nat Immunol 7:333-337

6. Arai F, Hirao A, Ohmura M, Sato H, Matsuoka S, Takubo K, Ito K, Koh GY, Suda T 2004 Tie2/angiopoietin-1 signaling regulates hematopoietic stem cell quiescence in the bone marrow niche. Cell 118:149-161

7. Kiel MJ, Yilmaz OH, Iwashita T, Yilmaz OH, Terhorst C, Morrison SJ 2005 SLAM family receptors distinguish hematopoietic stem and progenitor cells and reveal endothelial niches for stem cells. Cell 121:1109-1121

8. Kopp HG, Avecilla ST, Hooper AT, Rafii S 2005 The bone marrow vascular niche: home of HSC differentiation and mobilization. Physiology (Bethesda) 20:349-356

9. Arai F, Suda T 2007 Maintenance of quiescent hematopoietic stem cells in the osteoblastic niche. Ann N Y Acad Sci 1106:41-53

10. Ueda T, Tsuji K, Yoshino H, Ebihara Y, Yagasaki H, Hisakawa H, Mitsui T, Manabe A, Tanaka R, Kobayashi K, Ito M, Yasukawa K, Nakahata T 2000 Expansion of human NOD/SCID-repopulating cells by stem cell factor, Flk2/Flt3 ligand, thrombopoietin, IL-6, and soluble IL-6 receptor. J Clin Invest 105:1013-1021

11. Madkaikar M, Ghosh K, Gupta M, Swaminathan S, Mohanty D 2007 Ex vivo expansion of umbilical cord blood stem cells using different combinations of cytokines and stromal cells. Acta Haematol 118:153-159

12. Xu MJ, Tsuji K, Ueda T, Mukouyama YS, Hara T, Yang FC, Ebihara Y, Matsuoka S, Manabe A, Kikuchi A, Ito M, Miyajima A, Nakahata T 1998 Stimulation of mouse and human primitive hematopoiesis by murine embryonic aorta-gonadmesonephros-derived stromal cell lines. Blood 92:2032-2040

13. Taichman RS, Reilly MJ, Emerson SG 1996 Human osteoblasts support human hematopoietic progenitor cells in vitro bone marrow cultures. Blood 87:518-524

14. Chua KN, Chai C, Lee PC, Ramakrishna S, Leong KW, Mao HQ 2007 Functional nanofiber scaffolds with different spacers modulate adhesion and expansion of cryopreserved umbilical cord blood hematopoietic stem/progenitor cells. Exp Hematol 35:771-781

15. Nichols JE, Cortiella J, Lee J, Niles JA, Cuddihy M, Wang S, Bielitzki J, Cantu A, Mlcak R, Valdivia E, Yancy R, McClure ML, Kotov NA 2009 In vitro analog of human bone marrow from $3 \mathrm{D}$ scaffolds with biomimetic inverted colloidal crystal geometry. Biomaterials 30:1071-1079

16. Ogawa R, Mizuno H, Hyakusoku H, Shimada T 2006 Bone marrow regeneration using adipose-derived stem cells. J Nippon Med Sch 73:45-47 
17. Arinzeh TL, Tran T, McAlary J, Daculsi G 2005 A comparative study of biphasic calcium phosphate ceramics for human mesenchymal stem-cell-induced bone formation. Biomaterials 26:3631-3638

18. Hasegawa S, Neo M, Tamura J, Fujibayashi S, Takemoto M, Shikinami Y, Okazaki K, Nakamura T 2007 In vivo evaluation of a porous hydroxyapatite/poly-DL-lactide composite for bone tissue engineering. J Biomed Mater Res A 81:930-938

19. Hasegawa S, Tamura J, Neo M, Goto K, Shikinami Y, Saito M, Kita M, Nakamura T 2005 In vivo evaluation of a porous hydroxyapatite/poly-DL-lactide composite for use as a bone substitute. J Biomed Mater Res A 75:567-579

20. Sacchetti B, Funari A, Michienzi S, Di Cesare S, Piersanti S, Saggio I, Tagliafico E, Ferrari S, Robey PG, Riminucci M, Bianco P 2007 Self-renewing osteoprogenitors in bone marrow sinusoids can organize a hematopoietic microenvironment. Cell 131:324-336

21. Sugiyama O, Orimo H, Suzuki S, Yamashita K, Ito H, Shimada T 2003 Bone formation following transplantation of genetically modified primary bone marrow stromal cells. J Orthop Res 21:630-637

22. Ito Y, Tanaka N, Fujimoto Y, Yasunaga Y, Ishida O, Agung M, Ochi M 2004 Bone formation using novel interconnected porous calcium hydroxyapatite ceramic hybridized with cultured marrow stromal stem cells derived from Green rat. J Biomed Mater Res A 69:454-461
23. Hanawa H, Hematti P, Keyvanfar K, Metzger ME, Krouse A, Donahue RE, Kepes S, Gray J, Dunbar CE, Persons DA, Nienhuis AW 2004 Efficient gene transfer into rhesus repopulating hematopoietic stem cells using a simian immunodeficiency virus-based lentiviral vector system. Blood 103:4062-4069

24. Cao YA, Wagers AJ, Beilhack A, Dusich J, Bachmann MH, Negrin RS, Weissman IL, Contag CH 2004 Shifting foci of hematopoiesis during reconstitution from single stem cells. Proc Natl Acad Sci USA 101:221-226

25. Kiel MJ, Morrison SJ 2008 Uncertainty in the niches that maintain haematopoietic stem cells. Nat Rev Immunol 8:290-301

26. Adams GB, Chabner KT, Alley IR, Olson DP, Szczepiorkowski ZM, Poznansky MC, Kos CH, Pollak MR, Brown EM, Scadden DT 2006 Stem cell engraftment at the endosteal niche is specified by the calcium-sensing receptor. Nature 439:599603

27. Avecilla ST, Hattori K, Heissig B, Tejada R, Liao F, Shido K, Jin DK, Dias S, Zhang F, Hartman TE, Hackett NR, Crystal RG, Witte L, Hicklin DJ, Bohlen P, Eaton D, Lyden D, de Sauvage F, Rafii S 2004 Chemokine-mediated interaction of hematopoietic progenitors with the bone marrow vascular niche is required for thrombopoiesis. Nat Med 10:64-71 